\title{
THE ANNULAR HULL THEOREMS FOR THE KINEMATIC DYNAMO OPERATOR FOR AN IDEALLY CONDUCTING FLUID
}

\author{
C. CHICONE, Y. LATUSHKIN, AND S. MONTGOMERY-SMITH
}

\begin{abstract}
The group generated by the kinematic dynamo operator in the space of continuous divergence-free sections of the tangent bundle of a smooth manifold is studied. As shown in previous work, if the underlying Eulerian flow is aperiodic, then the spectrum of this group is obtained from the spectrum of its generator by exponentiation, but this result does not hold for flows with an open set of periodic trajectories. In the present paper, we consider Eulerian vector fields with periodic trajectories and prove the following annular hull theorems: The spectrum of the group belongs to the annular hull of the exponent of the spectrum of the kinematic dynamo operator, that is to the union of all circles centered at the origin and intersecting this set. Also, the annular hull of the spectrum of the group on the space of divergence free vector fields coincides with the smallest annulus, containing the spectrum of the group on the space of all continuous vector fields. As a corollary, the spectral abscissa of the generator coincides with the growth bound for the group.
\end{abstract}

\section{INTRODUCTION}

In the present paper we continue the study, begun in [4], of the spectral properties of the group generated by the kinematic dynamo operator for an ideally conducting fluid.

Date: June 16, 2001.

1991 Mathematics Subject Classification. 76W05, 58F99, 58G25.

Key words and phrases. magnetohydrodynamics, ideally conducting fluid, operator semigroups, spectral mapping theorem, weighted composition operators.

carmen@chicone.cs.missouri.edu supported in part by the NSF grant DMS9303767.

mathyl@mizzou1.missouri.edu supported in part by the NSF grant DMS9400518.

stephen@mont.cs.missouri.edu supported in part by the NSF grant DMS9201357. 
Throughout the paper $X$ denotes a smooth compact $n$-dimensional, $n \geq 3$, Riemannian manifold without boundary, $\mathcal{T} X$ denotes the tangent bundle of $X$, and $\mathcal{T}_{x} X$ denote the tangent space at $x \in X$. Also, $\mathbf{C}=C(X, \mathcal{T} X)$ denotes the Banach space of continuous sections of $\mathcal{T} X$ with sup-norm and $\mathbf{C}_{0}=C_{N D}(X, \mathcal{T} X)$ denotes the space of divergence free vector fields on $X$ taken either as the closure in $\mathbf{C}$ of the set of $C^{\infty}$-smooth vector fields $\mathbf{B}$ on $X$ with $\operatorname{div} \mathbf{B}=0$ or as the set of $\mathbf{B} \in \mathbf{C}$ orthogonal to all grad $g$ for $g$ a $C^{\infty}$-smooth real functions on $X$. The orthogonality here is understood with respect to a Riemannian metric and volume on $X$.

For an operator $A$ on a Banach space $E$, we use the notation $\sigma(A)=$ $\sigma(A, E)$ for the spectrum of $A$ on $E$. Also, for a set $S \subset \mathbb{C}$ we define $\mathcal{H}(S)$, the annular hull of $S$, to be the union of all circles centered at the origin of the complex plane that intersect $S$.

Let $u$ denote a smooth divergence-free vector field on $X$ and $\phi^{t}$ the flow generated by $u$. If for each $x \in X$ and $t \in \mathbb{R}$ there is an operator $\Phi(x, t): \mathcal{T}_{x} X \rightarrow \mathcal{T}_{\phi^{t} x} X$ such that $\Phi$ is smoothly dependent on both its arguments and if, for all $x \in X$ and $t, \tau \in \mathbb{R}$,

$$
\Phi(x, t+\tau)=\Phi\left(\phi^{t} x, \tau\right) \Phi(x, t), \quad \Phi(x, 0)=I,
$$

then $\Phi$ is called a smooth cocycle over $\phi^{t}$. A cocycle $\Phi$ induces on $\mathbf{C}$ a strongly continuous group $\left\{T^{t}\right\}$ defined by

$$
T^{t} \mathbf{B}(x):=\Phi\left(\phi^{-t} x, t\right) \mathbf{B}\left(\phi^{-t} x\right), \quad t \in \mathbb{R}, \quad x \in X, \quad \mathbf{B} \in \mathbf{C} .
$$

We let $L$ denote the infinitesimal generator of this group, $L \mathbf{B}=\left.\frac{d}{d t} T^{t} \mathbf{B}\right|_{t=0}$, so that $T^{t}=e^{t L}$. Also, for the remainder of the paper we will assume that the group $T^{t}$ preserves $\mathbf{C}_{0}$.

Our objective is to relate the spectra of $T^{t}$ and $L$ on $\mathbf{C}_{0}$. However, before stating the precise results we will prove, we pause to explain the relationship of $L$ to the kinematic dynamo operator for an ideally conducting fluid. For this, consider a steady incompressible conducting Eulerian fluid with velocity $u=u(x)$, for $x \in \mathbb{R}^{3}$, and let $\phi^{t}$ denote the corresponding flow. The induction equation for a magnetic field $\mathbf{H}=\mathbf{H}(x, t)$ is given by

$$
\dot{\mathbf{H}}=\nabla \times(u \times \mathbf{H})+\varepsilon \Delta \mathbf{H}, \quad \operatorname{div} \mathbf{H}=0,
$$

where $\varepsilon=\mathcal{R} e_{m}{ }^{-1}$, and $\mathcal{R} e_{m}$ is the magnetic Reynolds number (see, e.g., [16, Ch. 6]). Recently, the spectral properties of the kinematic dynamo operator $\mathcal{L}_{\varepsilon}$, defined by the right hand side of (2), have been the subject of intensive study [6,10,20,22], especially in connection with the famous "fast" dynamo problem, see $[1,2,3,7,22]$ and the references therein. 
For the ideally conducting fluid, $\varepsilon=0$, the system (2) given by

$$
\dot{\mathbf{H}}=-(u, \nabla) \mathbf{H}+(\mathbf{H}, \nabla) u, \quad \operatorname{div} \mathbf{H}=0,
$$

has [16] so-called Alfven solutions

$$
\mathbf{H}(x, t)=D \phi^{t}\left(\phi^{-t} x\right) \mathbf{B}\left(\phi^{-t} x\right), \quad \mathbf{H}(x, 0)=\mathbf{B}(x),
$$

where $D \phi^{t}(x)$ denotes the differential of $\phi^{t}$. These solutions are given by the group of "push-forward operators" induced, as in (1), by the cocycle $\Phi(x, t)=D \phi^{t}(x)$. The infinitesimal generator $\mathcal{L}=\mathcal{L}_{0}$ of this group is given by Lie differentiation in the direction of $u$, that is,

$$
\mathcal{L}: \mathbf{B} \mapsto(\mathbf{B}, \nabla) u-(u, \nabla) \mathbf{B}, \quad \operatorname{div} \mathbf{B}=0 .
$$

For $\varepsilon>0$, the operator $\mathcal{L}_{\varepsilon}=\varepsilon \Delta+\mathcal{L}$ is strongly elliptic and generates an analytic semigroup. This implies (see, e.g., [21]) that the spectrum of the group $e^{t \mathcal{L}_{\varepsilon}}$ is obtained from $\sigma\left(\mathcal{L}_{\varepsilon}\right)$ by exponentiation, in fact, $\sigma\left(e^{t \mathcal{L}_{\varepsilon}}\right)=\exp t \sigma\left(\mathcal{L}_{\varepsilon}\right)$ for $t \neq 0$. The operator $\mathcal{L}=\mathcal{L}_{0}$ is not elliptic and, as we will show later, this spectral mapping result does not hold for $\mathcal{L}$ with no additional assumptions on $u$.

For a general flow on a compact manifold $X$, we note that the operators in (1) belong to the class of so-called weighted composition operators, see $[8,13,14]$ for connections with dynamical system theory, and $[11,12]$ for connections with the theory of operator semigroups on Banach spaces. The semigroup (1) in the space of all continuous vector fields was studied in [5, 9] where the spectral mapping theorem and the annular hull theorem (see precise statements below) were proved. However, the proofs of these results for (1) on the space of divergence-free vector-fields requires a new technique, developed in [4] and in the present paper. This work was inspired by the important paper [6] which initiated recent research on the spectral properties of the groups (1) and (4) on the space of divergence-free vector-fields.

For notational convenience, for the semigroup $\left\{T^{t}\right\}$ in (1) we define $T:=T^{1}$. Also, we let $\mathcal{H}_{C}$ denote the smallest annulus, centered at the origin of the complex plane, that contains $\sigma(T, \mathbf{C})$.

The following Spectral Mapping Theorem was proved in [4] under the additional assumption that $u$ is a nonvanishing vector field.

Theorem 1. Suppose the aperiodic trajectories of the flow $\phi^{t}$ are dense in $X$. If $L$ is the infinitesimal generator of a group $\left\{T^{t}\right\}, T^{t}=e^{t L}$, of weighted composition operators, then

$$
\sigma\left(e^{t L}, \mathbf{C}_{0}\right)=\exp t \sigma\left(L, \mathbf{C}_{0}\right), \quad t \neq 0
$$


Moreover, $\sigma\left(T^{t}, \mathbf{C}_{0}\right)$ is invariant with respect to rotations centered at origin, and $\sigma\left(L, \mathbf{C}_{0}\right)$ is invariant with respect to translations along the imaginary axis. Also, $\sigma\left(T^{t}, \mathbf{C}_{0}\right)=\mathcal{H}_{C}$.

Below (cf. also [5]), we will give an example to show the spectral mapping theorem does not hold in general if there is an open set of periodic points. Also, the spectra of $T^{t}$ and $L$, generally, do not have the invariance property in this case. The main results of this paper are the following Annular Hull Theorems.

Theorem 2. Suppose $\phi^{t}$ is the flow of a smooth divergence free vector field on $X$. If $L$ is the infinitesimal generator of a group $\left\{T^{t}\right\}$ of weighted composition operators as in (1), then

$$
e^{t \sigma\left(L, \mathbf{C}_{0}\right)} \subset \sigma\left(e^{t L}, \mathbf{C}_{0}\right) \subset \mathcal{H}\left(e^{t \sigma\left(L, \mathbf{C}_{0}\right)}\right) .
$$

We note that $\mathcal{H}(\sigma(T, \mathbf{C}))$, generally, consists of several annuli centered at the origin. Passing to the subspace $\mathbf{C}_{0}$ radically changes the picture. In fact, $\mathcal{H}\left(\sigma\left(T, \mathbf{C}_{0}\right)\right)$ is exactly one annulus: the gaps in $\mathcal{H}(\sigma(T, \mathbf{C}))$, if any, are filled.

Theorem 3. If $\phi^{t}$ is the flow of a smooth divergence free vector field on $X$ and $\left\{T^{t}\right\}$ is a group fo weighted composition operators as in (1), then

$$
\mathcal{H}\left(\sigma\left(T, \mathbf{C}_{0}\right)\right)=\mathcal{H}_{C}
$$

We remark that there are nonnegative numbers $r_{ \pm}$such that $\mathcal{H}_{C}=$ $\left\{z \in \mathbb{C}: r_{-} \leq|z| \leq r_{+}\right\}$. Hence, $\mathcal{H}\left(\sigma\left(T, \mathbf{C}_{0}\right)\right)$ can be computed via exact Lyapunov-Oseledec exponents for the cocycle $\Phi$ with respect to all $\phi^{t}$-ergodic measures on $X$ (see $[4,13]$ for details).

We stress that for Theorem 2-3 the flow of the vector field $u$ is allowed to have rest points and periodic orbits and no assumption on the density of aperiodic trajectories is made. In the present paper we also show that Theorem 1 holds for a flow $\phi^{t}$ with rest points. We mention that, for a cocycle $\Phi$ in an infinite dimensional Banach space, the annular hull theorem, generally, fails $[13,18]$.

Recall the spectral abscissa $s$ and growth bound $\omega$ of the group $\left\{e^{t L}\right\}$ are defined by

$$
s(L):=\sup \{\operatorname{Re} z: z \in \sigma(L)\} \quad \text { and } \quad \omega(L):=\lim _{t \rightarrow \infty} t^{-1} \ln \left\|e^{t L}\right\| .
$$

See [19] for a detailed discussion of these concepts and for examples of groups with $s$ strictly less than $\omega$. The Annular Hull Theorems imply the following:

Corollary 4. If the hypotheses of Theorem 2 hold, then $s(L)=\omega(L)$. 
Of course, Theorems 1-3 and Corollary 4 apply to the group $\left\{e^{t \mathcal{L}}\right\}$. Since the spectral mapping theorem is valid for the semigroup generated by the kinematic dynamo operator $\mathcal{L}_{\varepsilon}$ with $\varepsilon>0$, one has $s\left(\mathcal{L}_{\varepsilon}\right)=\omega\left(\mathcal{L}_{\varepsilon}\right)$ for $\varepsilon>0$. For $\mathcal{L}_{0}=\mathcal{L}$, Corollary 4 shows that this equality is also valid for $\varepsilon=0$.

This observation might be useful in connection with the so-called "fast" dynamo problem. Recall (see, e.g., $[1,2,3])$ that the kinematic dynamo is called fast provided the limit $\limsup _{\varepsilon \rightarrow 0} \omega\left(\mathcal{L}_{\varepsilon}\right)$ is positive. M. Vishik [22] has shown that

$$
\limsup _{\varepsilon \rightarrow 0} \omega\left(\mathcal{L}_{\varepsilon}\right) \leq \omega\left(\mathcal{L}_{0}\right) .
$$

Thus, by the remarks above, his theorem can be reformulated to state

$$
\limsup _{\varepsilon \rightarrow 0} s\left(\mathcal{L}_{\varepsilon}\right) \leq s\left(\mathcal{L}_{0}\right)
$$

We stress that the last assertion does not involve the construction of the group $\left\{e^{t \mathcal{L}_{\varepsilon}}\right\}$; it is given in the terms of generators only.

M. Vishik [22] also proved that the dynamo is not fast provided the supremum over all Lyapunov numbers of the cocycle $D \phi^{t}$ is nonpositive (in fact, $\omega\left(\mathcal{L}_{0}\right)$ is equal to this supremum). We remark, that this condition for the absence of the fast dynamo remains valid even if this supremum is taken over all exact Lyapunov-Oseledec exponents for the cocycle $D \phi^{t}$ with respect to all $\phi^{t}$-ergodic measures on $X$. This remark holds with no restrictions on $u$ (see [4, Remark 3.8] for the case when $\phi^{t}$ has a dense set of aperiodic trajectories).

\section{Annular Hull Theorems}

In this section we will prove the Annular Hull Theorems on $\mathbf{C}_{0}$.

2.1. Preliminaries. Let $\sigma_{a p}(A)=\sigma_{a p}(A, E)$ denote the approximate point spectrum of the operator $A$ on a Banach space $E$, that is, the set of approximate eigenvalues $\lambda$ such that for every $\epsilon>0$ there exists an approximate eigenvector $\mathbf{B}$ with $\|(A-\lambda I) \mathbf{B}\| \leq \epsilon\|\mathbf{B}\|$.

Since $\mathbf{C}_{0}$ is a $T^{t}$-invariant subspace of $\mathbf{C}$, one has $\sigma_{a p}\left(T, \mathbf{C}_{0}\right) \subset$ $\sigma_{a p}(T, \mathbf{C})$. Using standard rescalings [19, p. 14], the Spectral Inclusion Theorem $\exp t \sigma_{a p}(L) \subset \sigma_{a p}\left(e^{t L}\right)$, and the spectral mapping theorems for point and residual spectra, see [19, pp. 84-85], Theorem 2 for $T=T^{1}$ as in (1) can be derived from the following fact.

Theorem 5. If $1 \in \sigma_{a p}(T, \mathbf{C})$, then $\sigma_{a p}\left(L, \mathbf{C}_{0}\right) \cap i \mathbb{R} \neq \emptyset$.

The proof of Theorem 5 is based on several lemmas. The first lemma is an adaptation of a result of $\mathrm{R}$. Manẽ [15]. 
Lemma 1. For $1 \in \sigma_{a p}(T, \mathbf{C})$ it is necessary and sufficient that there is a point $\bar{x} \in X$ and a vector $\bar{v} \in \mathcal{T}_{\bar{x}} X$, so that $\|\bar{v}\|=1$ and

$$
\sup _{t \in \mathbb{R}}\|\Phi(\bar{x}, t) \bar{v}\|<\infty .
$$

Proof. The proof of sufficiency is in [15]. We will prove the necessity.

Since $1 \in \sigma_{a p}(T, \mathbf{C})$, there is, for each natural number $N$ (see, e.g., [4, Lemma 2.6]) a section $f_{N} \in \mathbf{C}$ with $\left\|f_{N}\right\|_{\mathbf{C}}=1$ so that $\left\|T^{k} f_{N}\right\|_{\mathbf{C}} \leq 2$ for $|k| \leq N$. Choose $x_{N} \in X$ so that $\left\|f\left(x_{N}\right)\right\|=\left\|f_{N}\right\|_{\mathbf{C}}=1$, and define $v_{N}=f\left(x_{N}\right)$. Then, we have

$$
\left\|\Phi\left(x_{N}, k\right) v_{N}\right\| \leq\left\|T^{k} f_{N}\right\|_{\mathbf{C}} \leq 2 \text { for }|k| \leq N
$$

Since $X$ and the unit sphere in $\mathbb{R}^{n}$ are compact, we may assume that $\left(x_{N}, v_{N}\right) \rightarrow(\bar{x}, \bar{v})$ for some $\bar{x} \in X,\|\bar{v}\|=1$. We claim that (7) holds for $(\bar{x}, \bar{v})$. Indeed, for fixed $k \in \mathbb{Z}$, one has $\|\Phi(\bar{x}, k) \bar{v}\|=$ $\lim _{N \rightarrow \infty}\left\|\Phi\left(x_{N}, k\right) v_{N}\right\| \leq 2$ and (7) follows from the estimate

$$
\sup _{t \in \mathbb{R}}\|\Phi(\bar{x}, t) \bar{v}\| \leq \sup _{k \in \mathbb{Z}} \sup _{\tau \in[0,1]}\left\|\Phi\left(\phi^{k} \bar{x}, \tau\right) \Phi(\bar{x}, k) \bar{v}\right\| .
$$

Definition 6. A point $\bar{x}$, respectively a vector $\bar{v}$, that satisfies (7) is called a Manẽ point, respectively a Manẽ vector.

Define the prime period of a point $x \in X$ by $P(x)=\inf \left\{t>0: \phi^{t} x=\right.$ $x$ \} and note that $P$ is a lower semi-continuous function. Naturally, we assign $P(x)=\infty$ for aperiodic points $x \in X$. Suppose $\bar{x}$ is a Manẽ point. There are two cases to consider:

Case 1.: Uniformly bounded periods. There is a constant $\bar{P} \in \mathbb{R}_{+}$ and a neighborhood $U \ni \bar{x}$ such that $P(y) \leq \bar{P}$ for all $y \in U$.

Case 2.: Unbounded periods. For every $\bar{P} \in \mathbb{R}_{+}$and every neighborhood $U \ni \bar{x}$ there is an $x \in U$ such that $P(x) \geq \bar{P}$.

These cases will be considered in subsections 2.2 and 2.3, respectively. Our strategy in these subsections is as follows. Using the Manẽ point $\bar{x}$ and the Manẽ vector $\bar{v}$ as in (7), we will choose an appropriate point $x_{0} \in X$ near $\bar{x}$, and a vector $v \in \mathcal{T}_{x_{0}} X$. Then, we will define a divergence free vector field $w$, supported in a sufficiently small neighborhood $D$ of $x_{0}$, with values $v$ in a smaller neighborhood $B \subset D$ of $x_{0}$. We will construct a divergence-free approximate eigenvector $\mathbf{B}$ for $L$ that corresponds to a pure imaginary approximate eigenvalue $\lambda$ by integrating $T^{t} w(x)$ along sufficiently long segments of the trajectories of $\phi^{t}$ through the points $x \in D$. Finally, we will estimate $\|L \mathbf{B}-\lambda \mathbf{B}\|$ from above and $\|\mathbf{B}\|$ from below to show that $\mathbf{B}$ is indeed an approximate eigenvector for $L$. 
To construct $w$ as just described, we will use the following lemma from [4]:

Lemma 2. Suppose $x_{0} \in X$ is a point with $P\left(x_{0}\right)>0$, and $v \in \mathcal{T}_{x_{0}} X$. If numbers $\epsilon>0$ and $\delta>0$ are given, then there exist neighborhoods $B \subset D$ of $x_{0}$ with diam $D<\epsilon$ such that the following holds. There exists a smooth "bump"-function $\alpha: X \rightarrow[0,1]$ with $\alpha(x)=1$ for $x \in B$ and $\alpha(x)=0$ for $x \notin D$ and a continuously differentiable vector-field $w_{0}$ supported in $D \backslash B$, such that

(1) The vector-field $w(x)=\alpha(x) v+w_{0}(x)$ is divergence-free and has value $v$ in $B$;

(2) $\left\|w_{0}\right\|_{\mathbf{C}} \leq \delta$.

Proof. See [4, Lemma 2.3]. The geometrical idea of the proof is as follows. Take positive numbers $a<b<\epsilon$. There is a thin and long ellipsoid $B$ centered at $x_{0}$ whose longest axis directed along $v$ has length $b / 2$ while all its other axes have length $a / 2$. The ellipsoid $B$ is contained in a longer ellipsoid $D$ whose two longest axes, directed, respectively, along $v$ and along some perpendicular to $v$, both have length $b$, while all its other axes have length $a$.

For a function $\alpha$ as in the statement of the lemma one can compensate for the nonzero divergence of $\alpha(\cdot) v$ by taking an appropriate vector field $w_{0}$ whose norm satisfies the estimate $\left\|w_{0}\right\| \leq C a / b$ for some constant $C>0$ that does not depend on $a$ or $b$. To see this, imagine a flow of fluid, leaking out from the top of a thin vertical pipe $B$, then slowly recirculating to the bottom of $B$ through the much wider pipe $D$. At any rate, the lemma is proved by taking $a / b$ sufficiently small.

To carry out the estimates for $\mathbf{B}$, mentioned above, we will need to control the sojourn time of a trajectory segment that passes through $D$ and $B$. To do this, we will use the following definitions. For an open set $U$ containing $x \in X$ and $s>0$, define

$$
\Theta_{s, U}(x)=\left\{t \in \mathbb{R}:|t| \leq s, \phi^{t} x \in U\right\}, \quad M_{s, U}(x)=\operatorname{mes} \Theta_{s, U}(x) .
$$

Lemma 3. Suppose $n=\operatorname{dim} X \geq 3$. There is a constant $K>0$ such that for every $x_{0} \in X$ with $P\left(x_{0}\right)>0$, every $s>0$ with $s<P\left(x_{0}\right) / 8$ and every neighborhood $V \ni x_{0}$ there exist open sets $B \subset D$ as in Lemma 2, $B \subset D \subset V$, such that for every $x \in X$ the following inequality holds:

$$
M_{s, D}(x) \leq K M_{s, B}\left(x_{0}\right) .
$$

Proof. Use the semicontinuity of $P$ to choose a neighborhood $U \subset V$ of $x_{0}$ so small, that $P(y) \geq P\left(x_{0}\right) / 2$ for all $y \in U$. We will look for 
$B \subset D \subset U$. Since $s \leq P(y) / 4$, for any $y \in U$ one has $\phi^{t} y \notin U$ provided $s<|t| \leq 2 s$ (see [4, Lemma 2.4] for the aperiodic and Lemma 4 below for the periodic $\left.x_{0}\right)$. Hence, $\Theta_{s, D}(y)=\Theta_{2 s, D}(y)$ for each $y \in D$. As in [4, Lemma 2.4] this proves that (9) holds for all $x \in X$, provided it holds for $x \in D$. The rest of the rigorous proof is exactly as in [4, Lemma 2.5] provided $s$ replaces $N$ in that lemma. The geometrical idea is as follows. For $x \in D$, denote by $l_{s, D}(x)$ the length of the segment of the trajectory $\left\{\phi^{t} x:|t| \leq s\right\} \cap D$, by $t_{D}^{\prime}$ the time between the moment when the segment of the trajectory through $x$ first enters $D$ and the moment when it last exits $D$, and by $t_{B}^{\prime \prime}$ the time between the moment when the segment of the trajectory through $x_{0}$ first enters $B$ and the moment when it first exits $B$. Clearly, $M_{s, D}(x) \leq t_{D}^{\prime}$ and $M_{s, B}\left(x_{0}\right) \geq t_{B}^{\prime \prime}$. By the Mean Value Theorem $l_{s, D}(x)=\bar{k}_{1} t_{D}^{\prime}$ and $l_{s, B}\left(x_{0}\right)=k_{2} t_{B}^{\prime \prime}$, where $k_{1} \approx k_{2} \approx\left\|u\left(x_{0}\right)\right\|$ for small $U$. Since $B$ and $D$ are ellipsoids, described in the proof of Lemma 2 above, one can prove that $l_{s, D}(x) / l_{s, B}\left(x_{0}\right)$ is bounded.

2.2. Case 1. Uniformly bounded periods. Consider the Manẽ point $\bar{x}$ as in (7) and let the open set $U \ni \bar{x}$ be as in Case 1. In particular, $\bar{x}$ is periodic. Using the lower semicontinuity of $P$ we can and will choose $U$ sufficiently small so that one of the following alternatives holds:

Subcase 1.1.: $P(x)=0$ for all $x \in U$;

Subcase 1.2.: $P(x)>0$ for all $x \in U$;

Subcase 1.3.: $P(\bar{x})=0$ but $P\left(x_{n}\right)>0$ for a sequence $x_{n} \rightarrow \bar{x}$, $x_{n} \in U$.

For Subcase 1.1, every $x \in U$ is a rest point: $\phi^{t} x=x$. Also, since $\Phi$ is a cocycle, $\Phi(x, t)$ is a group for every $x \in U$. Let $A(x)$ denote the matrix that generates this group: $\Phi(x, t)=e^{t A(x)}, t \in \mathbb{R}, x \in U$ and note that the matrix-valued function $A$ is continuous on $U$. Also, for $w \in \mathbf{C}_{0}$ with supp $w \subset U$, we have $L w(x)=A(x) w(x)$. Since, by (7), $\sigma\left(e^{t A(\bar{x})}\right) \cap \mathbb{T} \neq \emptyset$, the spectral mapping theorem for matrices implies there is $\xi \in \mathbb{R}$ so that $i \xi \in \sigma(A(\bar{x}))$. Define $x_{0}=\bar{x}$, choose $v$ with $\|v\|=1$ so that $\left(A\left(x_{0}\right)-i \xi\right) v=0$, and fix $N \in \mathbb{N}$. Also, choose $\epsilon>0$ and $\delta>0$ so small that for every neighborhood $V \ni x_{0}$ with $\operatorname{diam} V<\epsilon$ the following holds:

$\sup \{\|(A(x)-i \xi) v\|: x \in V\}<1 / 2 N, \quad V \subset U, \quad \delta \sup \{\|A(x)-i \xi\|: x \in U\}<1 / 2 N$.

Apply Lemma 2 for these $\epsilon$ and $\delta$ to find $B \subset D \subset U$. Take $w=$ $\alpha(\cdot) v+w_{0}$ as indicated in Lemma 2. Recall, that $\alpha(x) \leq 1, x \in X$. 
Then, using the estimate (2) of the lemma, we find that:

$$
\|(L-i \xi) w\|_{\mathbf{C}_{0}}=\sup _{x \in D}\|(A(x)-i \xi) w(x)\| \leq 1 / N .
$$

As a result, $i \xi \in \sigma_{a p}(L)$, and Theorem 5 is proved.

For Subcase 1.2, the neighborhood $U$ does not contain rest points. By the semicontinuity of $P$ we can and will assume that there is a number $p_{0}>0$ such that $P(x) \geq p_{0}$ for all $x \in U$. Moreover, we note that $\sigma(\Phi(\bar{x}, P(\bar{x}))) \cap \mathbb{T} \neq \emptyset$. Indeed, if $\phi^{P(\bar{x})} \bar{x}=\bar{x}$, then $\Phi(\bar{x}, k P(\bar{x}))=[\Phi(\bar{x}, P(\bar{x}))]^{k}$ for $k \in \mathbb{Z}$. Since, by (7), the sequence $\left\{\left\|[\Phi(\bar{x}, P(\bar{x}))]^{k} \bar{v}\right\|\right\}$ is bounded, the matrix $\Phi(\bar{x}, P(\bar{x}))$ can not be hyperbolic. For a notational convenience, denote $x_{0}=\bar{x}$. Thus, there is some $v \in \mathcal{T}_{x_{0}} X$ and $\xi \in \mathbb{R}$, so that

$$
\Phi\left(x_{0}, P\left(x_{0}\right)\right) v=e^{i \xi} v, \quad\|v\|=1 .
$$

We will use the choice of $x_{0}$ and $v$ as in (10) in what follows.

For Subcase 1.3, the Manẽ point $\bar{x}$ is a rest point and each $x \in U$ is a periodic point. We claim that $\Phi\left(x_{n}, P\left(x_{n}\right)\right)$ can not be hyperbolic. To see this, we assume that $x_{n}$ is hyperbolic. By the Stable Manifold Theorem (see, e.g., [8]), the periodic orbit through $x_{n} \in U$ has a stable or an unstable manifold, contrary to the fact that $U$ consists entirely of periodic orbits. For a nonhyperbolic $x_{n}$ we denote $x_{0}=x_{n}$, and select $\xi \in \mathbb{R}$ and $v$ as in (10).

Starting from the assumption that $1 \in \sigma_{a p}(T, \mathbf{C})$, we have found in each subcase, 1.2 and 1.3 , a periodic point $x_{0}$ with period $P\left(x_{0}\right)>0$, a neighborhood $U$ of $x_{0}$ consisting entirely of periodic points whose periods are uniformly bounded and separated from zero, as well as a number $\xi \in \mathbb{R}$ and a vector $v$ as in (10). We will prove that $\lambda:=$ $i \xi / P\left(x_{0}\right)$ is an approximate eigenvalue for $L$.

Define $p=P\left(x_{0}\right)$ and let $\mu_{1}>0$ be a constant such that

$$
P(y) \leq \mu_{1} p, \quad y \in U .
$$

Fix a natural number $N>\mu_{1} p+1$. In what follows we use the letter $\mu$ (resp., $\nu$ ) with subscripts to denote "big" (resp., "small") constants that do not depend on $N$. Select (small) constants $\nu_{i}<1, i=0, \ldots, 5$. The required values of these constants will be determined later. Define

$$
\mu_{2}=2 \sup \left\{\left\|\Phi\left(x_{0}, t\right)\right\|:|t| \leq \mu_{1} p\right\}, \quad R=N+\mu_{1} p .
$$

Also, fix $s>0$ so that $s<\min (p / 8,1 / 4)$ and

$$
\max _{|t| \leq s}\left|e^{-\frac{i \xi t}{p}}-1\right| \leq \nu_{4},
$$




$$
\sup _{|t| \leq s}\left\|\Phi\left(\phi^{-t} x_{0}, t\right) v-v\right\| \cdot \sup _{|n| \leq 3 N / p}\left\|\left[\Phi\left(x_{0}, p\right)\right]^{n}\right\| \leq \nu_{2} .
$$

Lemma 4. There exists $\epsilon>0$ such that for every open set $D \ni x_{0}$ with $\operatorname{diam} D \leq \epsilon$ and every $y \in D$ the following implication holds:

$$
\text { If } \phi^{t} y \in D \text { and }|t| \leq R \text {, then } t \in \bigcup_{n \in \mathbb{Z}}(-s+n p, s+n p) \text {. }
$$

Proof. Suppose the lemma is false. Choose $D_{k} \ni x_{0}$ with $\operatorname{diam} D_{k} \rightarrow 0$ and $y_{k} \in D_{k}$ together with $t_{k} \in \mathbb{R},\left|t_{k}\right| \leq R$, so that $\phi^{t_{k}} y_{k} \in D_{k}$, but $t_{k} \notin \bigcup_{n \in \mathbb{Z}}(-s+n p, s+n p)$. By compactness, we may assume $t_{k} \rightarrow t^{*}$, $\left|t^{*}\right| \leq R$. By continuity, $\phi^{t_{k}} y_{k} \rightarrow \phi^{t^{*}} x_{0}$, and, therefore, $\phi^{t^{*}} x_{0}=x_{0}$. Since $t^{*}=n p+\tau$ for some $n \in \mathbb{Z}$ and some $\tau$ with $s<\tau<p-s$, one has $\phi^{\tau} x_{0}=x_{0}$. Since $p=P\left(x_{0}\right)$ is the prime period, in contradiction.

Fix $\epsilon>0$ as in Lemma 4. Choose a neighborhood $V \ni x_{0}$ with $\operatorname{diam} V<\epsilon$ so small that the following inequalities hold:

$$
\begin{array}{r}
\sup \left\{\|\Phi(y, t)\|:|t| \leq \mu_{1} p, y \in V\right\} \leq \mu_{2}, \\
\sup _{y \in V|t| \leq 3 N} \sup _{|t|}\left\|\Phi\left(\phi^{-t} y, t\right)-\Phi\left(\phi^{-t} x_{0}, t\right)\right\| \leq \nu_{1} .
\end{array}
$$

We note that $\Phi(x, 0)=I$, for $x \in X$. Fix $\delta$ so that

$$
\begin{aligned}
& \delta<\nu_{3}, \\
& \delta \max _{|t| \leq s}\left\|\Phi\left(\phi^{-t} x_{0}, t\right)\right\| \cdot \sup _{|n| \leq 3 N / p}\left\|\left[\Phi\left(x_{0}, p\right)\right]^{n}\right\| \leq \nu_{5} .
\end{aligned}
$$

Let $B \subset D, D \subset V$ be the open sets from Lemma 3 with the prescribed choice of $x_{0}, s, V, \epsilon$ and $\delta$. We define $m=M_{s, B}\left(x_{0}\right)$. Consider $\alpha$ and $w_{0}$ as indicated in Lemma 2. Finally, choose a smooth function $\gamma: \mathbb{R} \rightarrow[0,1]$ with $\operatorname{supp} \gamma \subset[-N, N]$ so that $\left|\gamma^{\prime}(t)\right| \leq 2 / N$ and

$$
\gamma(t) \geq \frac{1}{4} \text { for }|t| \leq \frac{N}{2}
$$

For $w=\alpha(\cdot) v+w_{0}$ as in Lemma 2, we define a vector field $\mathbf{B}$ as follows:

$$
\mathbf{B}(x)=\int_{-\infty}^{\infty} \gamma(t) e^{-\frac{i \xi t}{p}}\left(T^{t} w\right)(x) d t, \quad x \in X .
$$

Since $w$ is divergence-free, $\mathbf{B}$ also is divergence-free. We claim there is a constant $\mu>0$, independent of $N$, such that

$$
\left\|L \mathbf{B}-\frac{i \xi}{p} \mathbf{B}\right\|_{\mathbf{C}} \leq \frac{\mu}{N}\|\mathbf{B}\|_{\mathbf{C}} .
$$

The inequality (20) guarantees $\sigma_{a p}(L) \cap i \mathbb{R} \neq \emptyset$, and proves Theorem 5 . We prove (20) in two steps. 
Step 1. The Upper Estimate . We will show there is a constant $\mu>0$ such that, for $m$ defined above,

$$
\left\|L \mathbf{B}-\frac{i \xi}{p} \mathbf{B}\right\|_{\mathbf{C}} \leq \mu \cdot m .
$$

A direct calculation shows:

$$
(L \mathbf{B})(x)-\frac{i \xi}{p} \mathbf{B}(x)=-\int_{-\infty}^{\infty} \gamma^{\prime}(t) e^{-\frac{i \xi t}{p}}\left(T^{t} w\right)(x) d t .
$$

Recall that $w(x)=\alpha(x) v+w_{0}(x)$ and, for $x \in X$, define

$$
\begin{aligned}
& I(x)=\int_{-\infty}^{\infty}\left|\gamma^{\prime}(t)\right|\left\|\Phi\left(\phi^{-t} x, t\right) v\right\| \alpha\left(\phi^{-t} x\right) d t, \\
& J(x)=\int_{-\infty}^{\infty}\left|\gamma^{\prime}(t)\right|\left\|\Phi\left(\phi^{-t} x, t\right) w_{0}\left(\phi^{-t} x\right)\right\| d t .
\end{aligned}
$$

To prove (21) it suffices to show there is a constant $\mu>0$ such that, for all $x \in X$,

$$
\|I(x)\| \leq \mu \cdot m, \quad\|J(x)\| \leq \mu \cdot m .
$$

Clearly, for $x \notin \bigcup_{y \in D} \bigcup_{0 \leq t \leq P(y)} \phi^{t}(y)$, since $\alpha\left(\phi^{-t} x\right)=0$ and since $w\left(\phi^{-t}(x)\right)=0$, we have $I(x)=J(x)=0$. Fix $x \in \bigcup_{y \in D} \bigcup_{0 \leq t \leq P(y)} \phi^{t}(y)$ and select $y \in D$ so that $x=\phi^{\tau} y$. In accordance with (11) we will assume $0 \leq \tau \leq \mu_{1} p$.

To estimate $\|I(x)\|$ from above, we note first, that

$$
\|I(x)\| \leq \int_{-\infty}^{\infty}\left|\gamma^{\prime}(t+\tau)\right|\left\|\Phi\left(\phi^{-t} y, t+\tau\right) v\right\| \alpha\left(\phi^{-t} y\right) d t
$$

Since $\tau \leq \mu_{1} p$ and supp $\gamma^{\prime} \subset[-N, N]$, the integration is unchanged if restricted to $|t| \leq R=\mu_{1} p+N \leq 3 N$. Also, $y \in D \subset V$. We use (15) and (14) to obtain the following estimate:

$$
\begin{aligned}
\|I(x)\| & \leq \int_{-\infty}^{\infty}\left|\gamma^{\prime}(t+\tau)\right|\|\Phi(y, \tau)\|\left(\left\|\Phi\left(\phi^{-t} x_{0}, t\right) v\right\|\right. \\
& \left.+\left\|\Phi\left(\phi^{-t} y, t\right) v-\Phi\left(\phi^{-t} x_{0}, t\right) v\right\|\right) \alpha\left(\phi^{-t} y\right) d t \leq \mu_{2}\left(I_{1}(y)+\nu_{1} I_{2}(y)\right),
\end{aligned}
$$

where

$$
\begin{aligned}
& I_{1}(y)=\int_{-\infty}^{\infty}\left|\gamma^{\prime}(t+\tau)\right|\left\|\Phi\left(\phi^{-t} x_{0}, t\right) v\right\| \alpha\left(\phi^{-t} y\right) d t, \\
& I_{2}(y)=\int_{-\infty}^{\infty}\left|\gamma^{\prime}(t+\tau)\right| \alpha\left(\phi^{-t} y\right) d t .
\end{aligned}
$$

We use Lemma 4 to estimate $I_{1}(y)$ and $I_{2}(y)$. Since $y \in D$ and $\operatorname{supp} \alpha \subset D$, the integration in $I_{1}(y)$ and $I_{2}(y)$ is unchanged if restricted to $t \in \bigcup_{n \in \mathbb{Z}}(-s+n p, s+n p)$. Also, we have $\Phi\left(\phi^{-t} x_{0}, t+n p\right)=$ 
$\left[\Phi\left(x_{0}, p\right)\right]^{n} \Phi\left(\phi^{-t} x_{0}, t\right)$. As a result,

$$
I_{1}(y)=\sum_{n \in \mathbb{Z}} \int_{|t| \leq s}\left|\gamma^{\prime}(t+n p+\tau)\right|\left\|\left[\Phi\left(x_{0}, p\right)\right]^{n} \Phi\left(\phi^{-t} x_{0}, t\right) v\right\| \cdot \alpha\left(\phi^{-t-n p} y\right) d t
$$

Since supp $\gamma^{\prime} \subset[-N, N]$, the integral in the RHS of (25) is equal to zero for $|n|>2 N / p$. To see this, we recall that $N \geq \mu_{1} p+1$ and $s<1$. Since $|t| \leq s$ and $|\tau| \leq \mu_{1} p$ in (25), if $|n|>2 N / p$, then we have $|t+\tau+n p| \geq|n| p-s-\mu_{1} p \geq|n| p-N>N$. Therefore, $I_{1}(y)$ does not exceed

$$
\begin{aligned}
& \sum_{|n| \leq \frac{2 N}{p}} \int_{|t| \leq s}\left|\gamma^{\prime}(t+n p+\tau)\right|\left(\left\|\left[\Phi\left(x_{0}, p\right)\right]^{n} v\right\|+\right. \\
& \left.\left\|\left[\Phi\left(x_{0}, p\right)\right]^{n} \cdot\left[\Phi\left(\phi^{-t} x_{0}, t\right) v-v\right]\right\|\right) \alpha\left(\phi^{-t-n p} y\right) d t
\end{aligned}
$$

We use (10) and (13) to estimate the last expression to obtain

$$
I_{1}(y) \leq\left(1+\nu_{2}\right) \sum_{|n| \leq 2 N / p} \int_{|t| \leq s}\left|\gamma^{\prime}(t+n p+\tau)\right| \alpha\left(\phi^{-t-n p} y\right) d t
$$

Similarly, for $I_{2}(y)$, we have:

$$
I_{2}(y) \leq \sum_{|n| \leq 2 N / p} \int_{|t| \leq s}\left|\gamma^{\prime}(t+n p+\tau)\right| \alpha\left(\phi^{-t-n p} y\right) d t .
$$

Since supp $\alpha \subset D$, for each integer $n$, the integration in (26)-(27) can be restricted to $t \in \Theta_{s, D}\left(\phi^{-n p} y\right)$, see (8). Recall that, for $t \in \mathbb{R}$ and $x \in X$, we have $\left|\gamma^{\prime}(t)\right| \leq 2 / N$ and $\alpha(x) \leq 1$. As a result, there is a constant $\mu^{\prime}>0$ such that

$$
\|I(x)\| \leq \frac{\mu^{\prime}}{N} \sum_{|n| \leq \frac{2 N}{p}} M_{s, D}\left(\phi^{-n p} y\right) .
$$

Finally, we use estimate (9) with $x=\phi^{-n p} y$ to obtain the first inequality in (23):

$$
\|I(x)\| \leq \frac{\mu^{\prime}}{N} \cdot\left[\frac{2 N}{p}\right] \cdot K M_{s, B}\left(x_{0}\right) \leq \mu m,
$$

where the brackets denote the integer part.

To prove the second inequality in (23) we arrive, as in (25) above, to the following estimate:

$$
J(x) \leq \mu_{2}\left[J_{1}(y)+\nu_{1} J_{2}(y)\right]
$$


where

$$
\begin{aligned}
& J_{1}(y)=\int_{-\infty}^{\infty}\left|\gamma^{\prime}(t+\tau)\right|\left\|\Phi\left(\phi^{-t} x_{0}, t\right) w_{0}\left(\phi^{-t} y\right)\right\| d t \\
& J_{2}(y)=\int_{-\infty}^{\infty}\left|\gamma^{\prime}(t+\tau)\right|\left\|w_{0}\left(\phi^{-t} y\right)\right\| d t
\end{aligned}
$$

We use the estimate $\left\|w_{0}\right\|_{\mathbf{C}} \leq \delta$ from Lemma 2. Also, since $\gamma^{\prime}$ is supported in $[-N, N]$ and in view of Lemma 4 , the integration in $J_{1}(y)$ and $J_{2}(y)$ can be restricted as for $I_{1}(y)$ and $I_{2}(y)$ above, to $t \in \bigcup_{|n| \leq 2 N / p}(-s+n p, s+n p)$. Since supp $w_{0} \subset D$, as in (25)-(27) above, we use the estimates (17) and (16) to obtain

$$
\begin{aligned}
& J_{1}(y) \leq \nu_{5} \sum_{|n| \leq 2 N / p} \int_{|t| \leq s}\left|\gamma^{\prime}(t+n p+\tau)\right| \chi_{D}\left(\phi^{-t-n p} y\right) d t, \\
& J_{2}(y) \leq \nu_{3} \sum_{|n| \leq 2 N / p} \int_{|t| \leq s}\left|\gamma^{\prime}(t+n p+\tau)\right| \chi_{D}\left(\phi^{-t-n p} y\right) d t
\end{aligned}
$$

where $\chi_{D}(\cdot)$ is the characteristics function of $D$. Using (9), as in (29), we have for some positive constants $\mu^{\prime}$ and $\mu^{\prime \prime}$ :

$$
J_{1}(y) \leq \mu^{\prime} \cdot m, \quad J_{2}(y) \leq \mu^{\prime \prime} \cdot m .
$$

As a result, we obtain the second inequality in (23). Therefore, (21) is proved.

Step 2. The Lower Estimate. We will prove there is a constant $\nu>0$ such that

$$
\|\mathbf{B}\|_{\mathbf{C}} \geq\left\|\mathbf{B}\left(x_{0}\right)\right\| \geq \nu N m .
$$

This, together with (21), gives (20).

Define

$$
\begin{aligned}
& \tilde{I}\left(x_{0}\right)=\left\|\int_{-\infty}^{\infty} \gamma(t) e^{-\frac{i \xi t}{p}} \Phi\left(\phi^{-t} x_{0}, t\right) v \cdot \alpha\left(\phi^{-t} x_{0}\right) d t\right\|, \\
& \tilde{J}\left(x_{0}\right)=\left\|\int_{-\infty}^{\infty} \gamma(t) e^{-\frac{i \xi t}{p}} \Phi\left(\phi^{-t} x_{0}, t\right) w_{0}\left(\phi^{-t} x_{0}\right) d t\right\|
\end{aligned}
$$

and note that

$$
\left\|\mathbf{B}\left(x_{0}\right)\right\| \geq \tilde{I}\left(x_{0}\right)-\tilde{J}\left(x_{0}\right)
$$

Recall that $\operatorname{supp} \gamma \subset[-N, N]$ and that, for $t \in \operatorname{supp} \gamma$, we have $0 \leq$ $\gamma(t) \leq 1$. Using arguments similar to those employed in (32) and (25)-(29), only with the estimate $\left|\gamma^{\prime}(t)\right| \leq 2 / N$ replaced by $\gamma(t) \leq$ 1 , together with an estimate based on the estimate $\left\|w_{0}\right\|_{\mathbf{C}} \leq \delta$ from 
Lemma 2 and (17), we find there is a constant $\mu>0$ such that

$$
\begin{aligned}
\tilde{J}\left(x_{0}\right) & \leq \sum_{|n| \leq 2 N / p} \int_{|t| \leq s}|\gamma(t+n p)| \cdot\left\|\left[\Phi\left(x_{0}, p\right)\right]^{n}\right\| \cdot\left\|\Phi\left(\phi^{-t} x_{0}, t\right) w_{0}\left(\phi^{-t} x_{0}\right)\right\| d t \\
& \leq \nu_{5} \sum_{|n| \leq 2 N / p} \int_{|t| \leq s}|\gamma(t+n p)| \chi_{D}\left(\phi^{-t} x_{0}\right) d t \leq \mu \nu_{5} N m
\end{aligned}
$$

We use (10) to estimate $\tilde{I}\left(x_{0}\right)$ from below as follows:

$$
\begin{aligned}
\tilde{I}\left(x_{0}\right) & =\left\|\sum_{n \in \mathbb{Z}} \int_{|t| \leq s} \gamma(t+n p) e^{-\frac{i \xi t}{p}}\left[e^{-i \xi} \Phi\left(x_{0}, p\right)\right]^{n} \Phi\left(\phi^{-t} x_{0}, t\right) v \cdot \alpha\left(\phi^{-t} x_{0}\right) d t\right\| \\
& \geq \tilde{I}_{1}\left(x_{0}\right)-\tilde{I}_{2}\left(x_{0}\right)-\tilde{I}_{3}\left(x_{0}\right),
\end{aligned}
$$

where

$$
\begin{aligned}
& \tilde{I}_{1}\left(x_{0}\right)=\left\|\sum_{n \in \mathbb{Z}} \int_{|t| \leq s} \gamma(t+n p) v \cdot \alpha\left(\phi^{-t} x_{0}\right) d t\right\|, \\
& \tilde{I}_{2}\left(x_{0}\right)=\left\|\sum_{n \in \mathbb{Z}} \int_{|t| \leq s} \gamma(t+n p)\left(e^{-\frac{i \xi t}{p}}-1\right) v \cdot \alpha\left(\phi^{-t} x_{0}\right) d t\right\|, \\
& \tilde{I}_{3}\left(x_{0}\right)=\left\|\sum_{n \in \mathbb{Z}} \int_{|t| \leq s} \gamma(t+n p)\left[e^{-i \xi} \Phi\left(x_{0}, p\right)\right]^{n}\left(\Phi\left(\phi^{-t} x_{0}, t\right) v-v\right) \cdot \alpha\left(\phi^{-t} x_{0}\right) d t\right\| .
\end{aligned}
$$

We will estimate $\tilde{I}_{1}\left(x_{0}\right)$ from below. Recall (see (18)) that $\gamma(\tau) \geq \frac{1}{4}$ provided $|\tau| \leq \frac{N}{2}$. If $|n| \leq \frac{N-1}{2 p}$, then $|t+n p| \leq s+\frac{N-1}{2 p} \cdot p \leq \frac{N}{2}$. Hence, $\gamma(t+n p) \geq \frac{1}{4}$ for those $n$ and $|t| \leq s$. Also, $\|v\|=1$. Therefore,

$$
\tilde{I}_{1}\left(x_{0}\right)=\sum_{n \in \mathbb{Z}} \int_{|t| \leq s} \gamma(t+n p) \cdot \alpha\left(\phi^{-t} x_{0}\right) d t \geq \frac{1}{4} \sum_{|n| \leq \frac{N-1}{2 p}} \int_{|t| \leq s} \alpha\left(\phi^{-t} x_{0}\right) d t
$$

Since $\alpha\left(\phi^{-t} x_{0}\right)=1$ for $t \in \Theta_{s, B}\left(x_{0}\right)$, one has:

$$
\tilde{I}_{1}\left(x_{0}\right) \geq \frac{1}{4} \sum_{|n| \leq \frac{N-1}{2 p}} \int_{\Theta_{s, B}\left(x_{0}\right)} d t \geq \frac{1}{4} \cdot m \operatorname{card}\left\{n:|n| \leq \frac{N-1}{2 p}\right\} \geq \begin{array}{r}
N m \nu_{0} \\
(35)
\end{array}
$$

for some $\nu_{0}>0$.

As in (34), using (12), there is some $\mu^{\prime}>0$ such that

$$
\tilde{I}_{2}\left(x_{0}\right) \leq \nu_{4} \sum_{n \in \mathbb{Z}} \int_{|t| \leq s} \gamma(t+n p) \cdot \alpha\left(\phi^{-t} x_{0}\right) d t \leq \nu_{4} \mu^{\prime} N m .
$$


Similarly, using (13), we have

$$
\tilde{I}_{3}\left(x_{0}\right) \leq \nu_{2} \sum_{n \in \mathbb{Z}} \int_{|t| \leq s} \gamma(t+n p) \cdot \alpha\left(\phi^{-t} x_{0}\right) d t \leq \nu_{2} \mu^{\prime} N m .
$$

Combining (33)-(37) we obtain

$$
\left\|\mathbf{B}\left(x_{0}\right)\right\| \geq m N\left(\nu_{0}-\nu_{5} \mu-\nu_{2} \mu^{\prime}-\nu_{4} \mu^{\prime}\right) \geq \nu N m
$$

for a constant $\nu=\nu_{0}-\nu_{5} \mu-\nu_{2} \mu^{\prime}-\nu_{4} \mu^{\prime}>0$, provided $\nu_{2}, \nu_{4}, \nu_{5}$ are small enough.

We use (21) to obtain (20), and the theorem for Case 1 is proved.

2.3. Case 2. Unbounded periods. Assume that, for the Manẽ point $\bar{x}$ in $(7)$, the condition of Case 2 holds. Also, note that this condition always holds provided the set of aperiodic trajectories is dense in $X$. The latter situation was considered in [4]. However, the proof of Lemma 2.7 in [4] contains, in fact, the following assertion with no assumption about the density of aperiodic trajectories.

Lemma 5. If for every natural number $N$ there exist a point $x_{0}$, a vector $v \in \mathcal{T}_{x_{0}} X$ with $\|v\| \geq 1 / 2$, and an open set $U \ni x_{0}$ such that the following holds:

(a) $\sup \{\|\Phi(y, t) v\|: y \in U,|t| \leq 8 N\}<\mu$ for a constant $\mu$, independent on $N$;

(b) $P(y) \geq 8 N$ for all $y \in U$,

then $i \xi \in \sigma_{a p}(L)$ for every $\xi \in \mathbb{R}$.

Proof. We will indicate how to modify the proof of [4, Lemma 2.7] to obtain the lemma. Fix $\xi \in \mathbb{R}$ and $N$. Take $x_{0}, U$ and $v$ as indicated in the statement of the lemma. As in (12) and (13), there is a number $s^{\prime}>0$, so that for $|t| \leq s^{\prime}$,

$$
\left\|\Phi\left(\phi^{-t} x_{0}, t\right) v-v\right\| \text { and }\left|e^{-i \xi t}-1\right| \text { are small enough. }
$$

The conditions (38) must replace (2.18) and (2.19) in [4]. Define $\gamma$ : $\mathbb{R} \rightarrow[0,1]$ with $\left|\gamma^{\prime}(t)\right| \leq 2 / N$ and $\operatorname{supp} \gamma \subset[-N, N]$ such that $\gamma(t)=1$ for $|t| \leq s^{\prime}$. Choose $\delta$ so small that $\delta \max _{|t| \leq 2 N}\left\|T^{t}\right\|$ is small enough (cf. (16)-(17)). As in Lemma 4, use condition (b) to find an $\epsilon>0$ such that for every $V \ni x_{0}$ with $\operatorname{diam} V<\epsilon$ the segment of the trajectory $\left\{\phi^{t} y: s^{\prime} \leq|t| \leq N\right\}$ does not intersect $V$ whenever $y \in V$. Choose $V \ni x_{0}$ with $\operatorname{diam} V<\epsilon$ so that the LHS of (15) is small enough.

Apply Lemma 3 with $s=N$ and the prescribed choice of $V$. Choose $B \subset D, D \subset V$ as in Lemma 2 with the $\epsilon$ and $\delta$, selected above. For 
$w=\alpha(\cdot) v+w_{0}$ from Lemma 2, we define (cf. (19)):

$$
\mathbf{B}(x)=\int_{-\infty}^{\infty} e^{-i \xi t} \gamma(t) T^{t} w(x) d t
$$

As in (20) we show that $\|L \mathbf{B}-i \xi \mathbf{B}\|_{\mathbf{C}} \leq \frac{\mu}{N}\|\mathbf{B}\|_{\mathbf{C}}$ for a constant $\mu$, independent on $N$. To this end, we prove, that

$\|(L \mathbf{B})(x)-i \xi \mathbf{B}(x)\|=\left\|\int_{-\infty}^{\infty} e^{-i \xi t} \gamma^{\prime}(t) T^{t} w(x) d t\right\| \leq \frac{\mu}{N} M_{N, B}\left(x_{0}\right), \quad x \in X$,

for some $\mu>0$, and $\left\|\mathbf{B}\left(x_{0}\right)\right\| \geq \nu M_{N, B}\left(x_{0}\right)$ for some $\nu>0$.

The arguments are similar (and easier) than the arguments in Step 1 and Step 2 above. We note that the integration in (39) is unchanged if restricted to $t \in \Theta_{N, D}(x)$. Also, $\mathbf{B}(x)=0$ provided $x \notin \cup_{|t| \leq 2 N} \phi^{t}(D)$. Besides, for $s^{\prime}$ as above, the segment of the trajectory $\left\{\phi^{t} y: s^{\prime} \leq|t| \leq\right.$ $N\}, y \in D$ does not intersect $D \subset V$. As a result, the segment of the trajectory $\left\{\phi^{t} x:|t| \leq 2 N\right\}, x \in X$ spends not more than time $2 s^{\prime}$ in $D$, and we do not need to replace the integral in (39) by a sum as in (25). To obtain the desired estimates, we use condition (a) similarly to (14) above. Finally, condition (a) combined with (38) is used in the estimates as in (13) and (15) above.

To finish the proof of Theorem 5, it remains to show that in Case 2 conditions (a) and (b) in Lemma 5 hold. We use (7) to define $\mu=$ $2 \sup \{\|\Phi(\bar{x}, t) \bar{v}\|: t \in \mathbb{R}\}$. Fix $N \in \mathbb{N}$. Let $\bar{P}=9 N, v=\bar{v}$, and use the continuity of $\Phi$ to find $U \ni \bar{x}$ such that (a) in Lemma 5 holds. Since we are in Case 2, for this $U$ and $\bar{P}$ there is a point $x_{0} \in U$ with $P\left(x_{0}\right) \geq 9 N$. The prime periods function $P$ is lower semicontinuous. Hence, we can find a smaller neighborhood $U^{\prime} \subset U$ of $x_{0}$, so that (b) in Lemma 5 holds. By Lemma $5 \sigma_{a p}\left(L, \mathbf{C}_{0}\right) \cap i \mathbb{R} \neq \emptyset$, and Theorem 5 for Case 2 is proved.

\subsection{Proof of Theorem 3. Recall that we must prove}

$$
\mathcal{H}\left(\sigma\left(T, \mathbf{C}_{0}\right)\right)=\mathcal{H}_{C},
$$

where $T=T^{1}$ and $\mathcal{H}_{C}=\left\{z \in \mathbb{C}: r_{-} \leq|z| \leq r_{+}\right\}$denotes the smallest annulus containing $\sigma(T, \mathbf{C})$. Using the notation $\operatorname{rs}(A)=\operatorname{rs}(A, E)$ to denote the spectral radius of an operator $A$ on a Banach space $E$, we clearly have

$$
r_{+}=\operatorname{rs}(T, \mathbf{C}), \quad r_{-}=\left(\operatorname{rs}\left(T^{-1}, \mathbf{C}\right)\right)^{-1}=\inf \{|\lambda|: \lambda \in \sigma(T, \mathbf{C})\} .
$$


Proof. Since $\sigma_{a p}\left(T, \mathbf{C}_{0}\right) \subset \sigma_{a p}(T, \mathbf{C})$, obviously, $\mathcal{H}\left(\sigma_{a p}\left(T, \mathbf{C}_{0}\right)\right) \subset \mathcal{H}\left(\sigma_{a p}(T, \mathbf{C})\right)$. We will show that, in fact,

$$
\mathcal{H}\left(\sigma_{a p}\left(T, \mathbf{C}_{0}\right)\right)=\mathcal{H}\left(\sigma_{a p}(T, \mathbf{C})\right)
$$

The hull $\mathcal{H}\left(\sigma_{a p}(T, \mathbf{C})\right)$ consists of a union of circles centered at the origin of the complex plane. If $\Gamma_{r}:=\{z \in \mathbb{C}:|z|=r\} \subset \mathcal{H}\left(\sigma_{a p}(T, \mathbf{C})\right)$, then there is a point $\lambda:=r e^{i \theta} \in \sigma_{a p}(T, \mathbf{C})$. Consider the group $\tilde{T}^{t}=$ $r^{-t} e^{-i \theta t} T^{t}$ generated by $\tilde{L}=-\ln r-i \theta+L$. Since $1 \in \sigma_{a p}(\tilde{T}, \mathbf{C})$, Theorem 5 applied to $\left\{\tilde{T}^{t}\right\}$ implies there is some $\xi \in \mathbb{R}$ such that

$$
i \xi \in \sigma_{a p}\left(\tilde{L}, \mathbf{C}_{0}\right)=-\ln r-i \theta+\sigma_{a p}\left(L, \mathbf{C}_{0}\right) .
$$

By the Spectral Inclusion Theorem for $\left\{T^{t}\right\}$ on $\mathbf{C}_{0}$, we have $r e^{i(\xi+\theta)} \in$ $\sigma_{a p}\left(T, \mathbf{C}_{0}\right)$, and $\Gamma_{r} \subset \mathcal{H}\left(\sigma_{a p}\left(T, \mathbf{C}_{0}\right)\right)$. This proves (41).

Since the boundary of $\sigma\left(T, \mathbf{C}_{0}\right)$ belongs to $\sigma_{a p}\left(T, \mathbf{C}_{0}\right)$, the inclusion $\mathcal{H}\left(\sigma\left(T, \mathbf{C}_{0}\right)\right) \subset \mathcal{H}_{C}$ in (40) follows directly from (41). To prove the inverse inclusion, suppose $\Gamma_{r} \subset \mathcal{H}_{C}$ but $\sigma\left(T, \mathbf{C}_{0}\right) \cap \Gamma_{r}=\emptyset$. By rescaling, we can and will assume that $r=1$, that is $r_{-}<1<r_{+}$and $\sigma\left(T, \mathbf{C}_{0}\right) \cap$ $\mathbb{T}=\emptyset$. Let $P_{0}$ denote the Riesz projection for the operator $T$ on $\mathbf{C}_{0}$ corresponding to the part of $\sigma\left(T, \mathbf{C}_{0}\right)$ that lies inside of the unit disc $\mathbb{D}$. We claim that both $\sigma\left(T, \mathbf{C}_{0}\right) \cap \mathbb{D}$ and $\sigma\left(T, \mathbf{C}_{0}\right) \cap(\mathbb{C} \backslash \mathbb{D})$ are nonempty. Indeed, since $r_{-}<1<r_{+}$, the set $\mathcal{H}\left(\sigma_{a p}(T, \mathbf{C})\right)$ contains a circle with radius greater than one and a circle with the radius less than one. By (41), $\mathcal{H}\left(\sigma_{a p}\left(T, \mathbf{C}_{0}\right)\right)$ also contains these circles. This proves the claim.

As in [4, Theorem 3.4], one can show that there is an extension of $P_{0}$ to the Riesz projection $\mathcal{P}$ for the operator $T$ on $\mathbf{C}$. It follows, see $[5,13,14]$, that this projector $\mathcal{P}$ has a form $\mathcal{P} \mathbf{B}(x)=P_{C}(x) \mathbf{B}(x)$, $\mathbf{B} \in \mathbf{C}$ where $P_{C}: X \rightarrow \operatorname{proj}\left(\mathcal{T}_{x} X\right)$ is a continuous projection-valued function. Since $\mathcal{P}=P_{0}$ on $\mathbf{C}_{0}$, multiplication by $P_{C}(\cdot)$ preserves the set of divergence-free vector fields. This, see [4, Theorem 3.4], leads to the fact that $P_{C}(x)$ is either the identity or the zero operator, and, as a result, the same is true for the operator $P_{0}$, in contradiction to the above claim.

To construct $\mathcal{P}$ on $\mathbf{C}$ one can use the proof of [4, Theorem 3.4]. The idea is to define $\mathcal{P}$ on a dense subset of $\mathbf{C}$ consisting of "step-sections" of the form $\mathbf{B}(\cdot)=\sum \rho_{k}(\cdot) v_{k}$, where $\left\{\rho_{k}\right\}$ is a partition of unity, and $v_{k}$ are given vectors. For each $k$ we use Lemma 2 to find $w_{k} \in \mathbf{C}_{0}$ such that $w_{k}(x)=v_{k}$ for $x \in \operatorname{supp} \rho_{k}$. We then define $\mathcal{P} \mathbf{B}=\sum \rho_{k} P_{0} w_{k}$. As in the proof of [4, Theorem 3.4], this definition does not depend on the choice of $w_{k}$ and, moreover, the projection $\mathcal{P}$ can be extended to a bounded linear operator on C. In fact, the Steps 1,2,4,5 of the cited proof go through with no assumption on density of the aperiodic trajectories of $\phi^{t}$. To modify Step 3 of the cited proof for the case 
when this assumption does not hold, we use the sufficiency part of Lemma 1 to conclude that $1 \in \sigma_{a p}(T, \mathbf{C})$ instead of $\mathbb{T} \subset \sigma_{a p}(T, \mathbf{C})$ as in [4, Theorem 3.4, Step 3]. By (41), this contradicts the hyperbolicity of $T$ on $\mathbf{C}_{0}$, as required in Step 3 of [4, Theorem 3.4].

2.5. Example. We give an example of an Eulerian vector-field $u$, so that the spectral mapping theorem for the corresponding group $\left\{T^{t}\right\}$ does not hold. For this, consider the three dimensional torus $\mathbb{T}^{3}$, viewed as $\mathbb{R}^{3} / \mathbb{Z}^{3}$ with coordinates $(x, y, z)$ in $\mathbb{R}^{3}$ modulo $2 \pi$, and recall Euler's equations are

$$
u_{t}+(u \cdot \nabla) u=-\frac{1}{\rho} \nabla \mathbf{p}, \quad \operatorname{div} u=0,
$$

where $\rho$ is the fluid density and $\mathbf{p}$ is the pressure. The constant vector field $u$ given by $u(x, y, z)=(1,0,0)$ together with a constant pressure provides a (steady state) solution for $(42)$. Define $\mathcal{L}$ to be the Lie derivative in the direction of $u$. We will show that the spectral mapping theorem, considered in the space $\mathbf{C}_{0}$ of divergence-free vector fields on $\mathbb{T}^{3}$, does not hold for the corresponding group $T^{t}=e^{t \mathcal{L}}$ generated by $\mathcal{L}$, and that $\sigma\left(T^{t}\right)$ and $\sigma(L)$ are not invariant with respect to arbitrary rotations and translations.

The tangent bundle of $\mathbb{T}^{3}$ is trivial, so we consider the elements of the Banach space $\mathbf{C}_{0}$ of divergence-free sections as maps $f: \mathbb{T}^{3} \rightarrow \mathbb{C}^{3}$. With this representation, we have

$$
(\mathcal{L} f)(x, y, z)=\frac{\partial}{\partial x} f(x, y, z), \quad\left(T^{t} f\right)(x)=f(x+t, y, z)
$$

where the operations are taken componentwise.

The spectrum of $\mathcal{L}$ is $\{i k: k \in \mathbb{Z}\}$. To see this, note first that $F(x, y, z):=\left(0, e^{i k x}, 0\right)$ is a divergence-free eigenfunction of $\mathcal{L}$ with eigenvalue $i k$. On the other hand, if $\lambda$ is not of the form $i k$, then $\mathcal{L}-\lambda I$ is invertible. In fact, the inverse is given by

$$
(\mathcal{L}-\lambda I)^{-1} g(x, y, z)=\frac{e^{2 \pi \lambda}}{1-e^{2 \pi \lambda}} \int_{0}^{2 \pi} e^{-\lambda s} g(s+x, y, z) d s .
$$

Thus, $\sigma(L)=i \xi+\sigma(L)$ does not hold for all $\xi \in \mathbb{R}$

Let $t$ denote a real number that is incommensurate with $2 \pi$. Then $\sigma\left(T^{t}\right)=\mathbb{T}$. This follows from the Spectral Inclusion Theorem and the fact that the spectrum of the bounded operator $T^{t}$ is closed. Since $\exp t \sigma(\mathcal{L})$ is not the whole circle, the spectral mapping theorem fails, as required. It is also easy to give a direct verification that $\sigma\left(T^{t}\right)=\mathbb{T}$. For this, choose a point $\lambda=e^{i \theta} \in \mathbb{T}$. If $\epsilon>0$, then there is a integer $k$ 
so that $\left|e^{i k t}-e^{i \theta}\right|<\epsilon$. But, for $F$ defined above,

$$
\left\|\left(T^{t} F\right)(x, y, z)-e^{i \theta} F(x, y, z)\right\|=\left|e^{i k t}-e^{i \theta}\right|<\epsilon .
$$

Thus, $e^{i \theta} \in \sigma_{a p}\left(T^{t}\right)$.

If $t / 2 \pi$ is a rational number $q / p, q \in \mathbb{Z}, p \in \mathbb{N}$, then $\sigma(T)=\{z \in \mathbb{C}$ : $\left.z^{p}=1\right\}$ and, as required, is not invariant with respect to all rotations centered at origin. However, $\mathcal{H}\left(\sigma\left(T^{t}\right)\right)=\mathbb{T}=\mathcal{H}(\exp t \sigma(L))$.

\section{REFERENCES}

[1] V. I. Arnold, Some remarks on the antidynamo theorem, Moscow University Mathem. Bull., 6 (1982) 50-57.

[2] V. I. Arnold, Ya. B. Zel'dovich, A. A. Rasumaikin, and D. D. Sokolov, Magnetic field in a stationary flow with stretching in Riemannian space, Sov. Phys. JETP, 54 (6) (1981) 1083-1086.

[3] B. J. Bayly and S. Childress, Fast-dynamo action in unsteady flows and maps in three dimensions, Phy. Rev. Let. 59 (14) (1987) 1573-1576.

[4] C. Chicone, Y. Latushkin, and S. Montgomery-Smith, The spectrum of the kinematic dynamic operator for an ideally conducting fluid, Comm. Math. Phys., to appear.

[5] C. Chicone and R. Swanson, Spectral theory for linearization of dynamical systems, J. Diff. Eqns., 40 (1981) 155-167.

[6] R. de la Llave, Hyperbolic dynamical systems and generation of magnetic fields by perfectly conducting fluids, Geophys. Astrophys. Fluid Dynamics, 73 (1993) 123-131.

[7] S. Friedlander and M. Vishik, Dynamo theory methods for hydrodynamic stability, J. Math. Pures Appl. 72 (1993) 145-180.

[8] M. Hirsch, C. Pugh, and M. Shub, Invariant Manifolds, Lect. Notes Math. 583 (1977).

[9] R. Johnson, Analyticity of spectral subbundles, J. Diff. Eqns., 35 (1980) 366-387.

[10] I. Klapper and L. S. Young, Rigorous bounds on the fast dynamo growth rate involving topological entropy, Preprint (1994) 1-30.

[11] Y. Latushkin and S. Montgomery-Smith, Lyapunov theorems for Banach spaces, Bull. AMS 31 (1) (1994) 44-49.

[12] Y. Latushkin and S. Montgomery-Smith, Evolutionary semigroups and Lyapunov theorems in Banach Spaces,

[13] Y. Latushkin and A. M. Stepin, Weighted composition operators and linear extensions of dynamical systems, Russian Math. Surveys, 46, no. 2, (1992) $95-165$.

[14] J. Mather, Characterization of Anosov diffeomorphisms, Indag. Math., 30 (1968) 479-483.

[15] R. Manẽ, Quasi-Anosov diffeomorphisms and hyperbolic manifolds, Trans. Amer. Math. Soc., 229 (1977) 351-370.

[16] H. K. Moffatt, Magnetic Field Generation in Electrically Conducting Fluids, Cambridge Univ. Press, Cambridge, 1978.

[17] S. A. Molchanov, A. A. Ruzmaikin, and D. D. Sokolov, Kinematic dynamo in random flow, Sov. Phys. Usp. 28 (4) (1985) 307-327. 
[18] S. Montgomery-Smith, Stability and dichotomy of positive semigroups on $L_{p}$, preprint.

[19] R. Nagel (ed.) One-parameter Semigroups of Positive Operators, Lect. Notes Math., 1184, Springer-Verlag, NY, 1986.

[20] M. Nuńẽz, Localized eigenmodes of the induction equation, SIAM J. Appl. Math., 54, no. 5 (1994) 1254-1267.

[21] A. Pazy, Semigroups of Linear Operators and Applications to Partial Differential Equations, Springer-Verlag, N.Y./Berlin, 1983.

[22] M. M. Vishik, Magnetic field generation by the motion of a highly conducting fluid, Geophys. Astrophys. Fluid Dynamics, 48 (1989) 151-167.

Department of Mathematics, University of Missouri, Columbia MO 65211 